\title{
A influência dos atributos educacionais dos cursos profissionalizantes na empregabilidade
}

\section{The influence of the educational attributes of vocational courses on employability}

\author{
Paulo Isnard ${ }^{\mathrm{a}}$, José Marcos Mesquita ${ }^{\mathrm{b}}$, Ellen Fialho ${ }^{\mathrm{c}}$ \\ ${ }^{a}$ Mestre em Modelagem Computacional. Doutorando em Sistemas Informação e Gestão do Conhecimento. FUMEC MG. ppis- \\ nard@yahoo.com.br

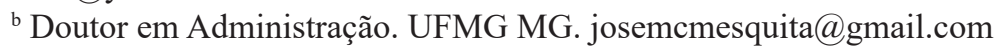 \\ ${ }^{c}$ Mestre em Sistemas Informação e Gestão do Conhecimento. FUMEC MG. ellenfialho@hotmail.com
}

\begin{abstract}
Resumo
Investigar os atributos para a empregabilidade é uma preocupação constante dentro da área acadêmica e corporativa e vem sofrendo mudanças constantes nas últimas décadas. Estudos mostram que apenas a formação profissional não garante a inserção de profissionais no mercado de trabalho. Este artigo investigou se e quanto as instituições de formação profissionalizante contribuem para aumentar as oportunidades de Empregabilidade do aluno, por meio do estabelecimento das relações entre as Competências Individuais, Marketing Educacional e Empregabilidade. O objetivo geral do artigo foi analisar a influência dos atributos educacionais das instituições de ensino profissional na empregabilidade, e os objetivos específicos foram: a) Identificar os atributos oferecidos pelos cursos profissionalizantes; b) Determinar as percepções dos alunos sobre os atributos educacionais; e c) Explicar a relação entre atributos educacionais e empregabilidade. Utilizou-se de uma pesquisa quantitativa descritiva, e os dados foram coletados por meio de um questionário respondido por alunos de instituição de ensino localizada em Minas Gerais. O tratamento estatístico foi baseado em modelagem de equações estruturais, utilizando o software PLS. Os principais resultados mostraram que o Plano Pedagógico e as Atividades Extras são capazes de influenciar positivamente a Empregabilidade, enquanto o Corpo Docente e Infraestrutura não se mostraram importantes.
\end{abstract}

Palavras-chave: Empregabilidade; Habilidades Individuais; Marketing Educacional; Cursos Profissionalizantes.

\begin{abstract}
Employability is a constant concern within the academic and investigating the attributes for corporate area and has been undergoing constant transformation in recent decades. Studies show that only vocational training does not guarantee the insertion of professionals in the labor market. This article investigated whether and how the vocational training institutions contribute to increase the employability opportunities of the student, through the establishment of relations between Individual Competencies, Educational Marketing and Employability. The general objective of the article was to analyze the influence of the educational attributes of professional education institutions on employability, and the specific objectives were: a) To identify the attributes offered by the vocational courses; b) Determine students' perceptions of educational attributes; and c) Explain the relationship between educational attributes and employability. Descriptive quantitative research was used, and the data were collected through a questionnaire answered by students from a teaching institution located in Minas Gerais. The statistical treatment was based on the modeling of structural equations using PLS software. The main results showed that the Pedagogical Plan and the Extra Activities are able to positively influence the Employability, while the Faculty and Infrastructure did not prove important.
\end{abstract}

Keywords: Employability; Individual Skills; Educational Marketing; Professional courses. 


\section{Introdução}

Formas cada vez mais inovadoras de gestão baseadas em tecnologia (Ma, Liu, Jiang, Yu, \& Gan, 2017) estão surgindo, o que gera um aumento na demanda por áreas específicas para o desempenho da atividade profissional qualificada (Moriset, 2019; Snihur \& Wiklund, 2018). A orientação do mercado de trabalho é buscar inovações, desta forma o trabalhador procurando a ascensão profissional, busca acompanhar a evolução dos requisitos profissionais por meio de formação profissional e, como o estudante, está se tornando a cada dia mais exigente.

Assim, a instituição de ensino deve estar preparada para analisar o desempenho do aluno que passa por um processo educacional de modo a verificar a percepção dele em relação aos serviços por elas prestados. A formação profissional por si só não garante a inserção do trabalhador, embora seja essencial para melhorar as condições de empregabilidade. A integração profissional é facilitada por meio de conhecimentos, habilidades e atitudes, desenvolvidos com base na formação educacional, focada nas necessidades do mercado (Lemos, Rodriguez, \& Monteiro, 2011; Lysova, Jansen, Khapova, Plomp, \& Tims, 2018; Zobel, 2017).

Cursos profissionalizantes e novas competências surgiram como potentes plataformas de empregabilidade. Os trabalhadores que procuram empregabilidade estão sendo obrigados a desenvolver capacidades que ultrapassem suas principais áreas, tornando-se multifuncionais, o que corrobora com uma tendência global de crescimento dos temas relacionados à interdisciplinaridade (Bernstein, Machlup, Polanyi, \& Shera, 2014; Choi \& Pak, 2006).

Isso fez com que o trabalhador se preocupasse constantemente com a busca de novas habilidades. Essa busca incessante pela aquisição de diferenciais, seja por meio da formação educacional ou pelo uso de experiências decorrentes do cotidiano, deve-se ao fato de as empresas estarem criando novos setores para atender novas demandas (M. Souza, Helal, \& Paiva, 2017).

Fatores causais que levam à empregabilidade permanecem especulativos. A partir do contexto descrito, o conceito de empregabilidade, usado neste artigo, é consolidado como a capacidade dos trabalhadores de se adaptarem às mudanças que as organizações estão experimentando.

O uso do termo empregabilidade refere-se não somente às habilidades específicas da profissão, mas também às habilidades ambicionadas em geral para um bom profissional. O trabalhador deve estar sempre em constante aprendizado, incluindo os aspectos tecnológicos que estão gradualmente substituindo mão de obra não especializada, notadamente em termos operacionais (Castells, 2007; Lemos \& Costa, 2012; Sudha, Samuel, \& Narkeesh, 2018).

A empregabilidade tem atraído considerável interesse desde os primeiros anos desta década, já que as tecnologias vêm substituindo a força de trabalho de maneira intensa. Uma das mais significativas características da empregabilidade atualmente é a discussão da influência dos cursos profissionalizantes.

Cursos profissionalizantes, especialmente aqueles que proporcionam treinamento, são importantes, pois permitem aos alunos aprimorar suas habilidades e adquirir capacidades específicas que às vezes não são abordadas nos cursos de graduação. O interesse por tais cursos no campo acadêmico culminou no aumento de publicações de estudos sobre empregabilidade (Van der Heijden et al., 2018).

$\mathrm{Na}$ economia global, as habilidades adquiridas se tornam uma questão central para empregabilidade. Com base nas mudanças impostas pela globalização, surgem novas ferramentas de gestão que acarretam impactos no processo administrativo e acadêmico das instituições de ensino. É urgente estudar e analisar a influência dos fatores ofertados nos cursos profissionalizantes na empregabilidade, principalmente pela falta 
de consenso sobre o que realmente determina o acesso ou ascensão dos alunos no atual mercado de trabalho (Kotler, 2017; Lemos, Rocha-Pinto, \& Dubeux, 2014).

Diante do exposto, a questão que norteia a pesquisa é: "Qual a influência dos atributos educacionais dos cursos profissionalizantes na empregabilidade"? Portanto, com a presente pesquisa objetiva-se analisar a influência dos atributos educacionais das instituições do ensino profissionalizantes na empregabilidade. Especificamente, pretende-se: a) Identificar os atributos oferecidos pelas escolas profissionalizantes; b) Identificar a percepção dos alunos quanto aos atributos educacionais; c) Avaliar a relação dos atributos educacionais com a empregabilidade.

O tema é atual porque propõe uma relação entre a empregabilidade e o desenvolvimento de habilidades contemporâneas requeridas no mercado de trabalho. Abordar a empregabilidade é um desafio, pois lidar com questões interdisciplinares é tratar com questões conflitantes.

No que diz respeito às publicações acadêmicas e científicas, há claramente uma lacuna. Na busca realizada em 09 de abril de 2018 na base de dados Scopus com as chaves "empregabilidade" e "curso profissional", foram obtidas 41 publicações, porém, ao pesquisar apenas "empregabilidade", foram encontrados 17.842 documentos. Assim, a pesquisa mostrou consistentemente uma lacuna acadêmica envolvendo empregabilidade, provocando assim interesse e curiosidade.

\section{Referencial Teórico}

Há um crescente corpo de literatura que reconhece a empregabilidade e sua interface com os atributos educacionais dos cursos profissionalizantes. Este referencial teórico abrange três temas principais, a saber: Empregabilidade, na qual se realiza uma contextualização histórica da empregabilidade e seus conceitos; Habilidades Individuais e empregabilidade; e Marketing Educacional, que retrata a influência do marketing na empregabilidade. Este trabalho abordou os temas mais relevantes para a consecução dos objetivos propostos.

E propõe uma interface entre os temas através da construção de hipóteses (Arraes, Cabral, Santos, Silva, \& Penha, 2017; Zanini, 2007), detalhadas à seguir.

\subsection{Empregabilidade}

A empregabilidade dos indivíduos depende de suas habilidades, nas quais eles fazem uma variedade de investimentos. A educação formal é um desses investimentos. Estudos mostram que mais níveis de escolaridade e ensino superior levam a mais sucesso no mercado de trabalho (Shukla \& Kumar, 2018). No entanto, embora os efeitos deste sucesso estejam bem documentados, é menos compreendido como esses efeitos surgem. Vários aspectos cognitivos e não cognitivos dificultam uma investigação mais aguda dos mecanismos próximos (Arraes et al., 2017).

Há evidências destacando a importância das habilidades cognitivas. Enquanto habilidades não cognitivas (postura, discernimento, uso de vocabulário adequado, etc.) são frequentemente usadas como um termo vago para qualquer habilidade que não é cognitiva (e, de fato, muitas habilidades geralmente chamadas de não-cognitivas contêm um forte componente cognitivo), uma literatura crescente (Stewart, 1998; Sveiby, 1998) investiga diferentes habilidades claramente especificadas empiricamente no domínio não-cognitivo.

Uma dimensão importante das habilidades não cognitivas são as habilidades sociais. Deming (2017) argumenta que a importância das habilidades sociais no mercado de trabalho está crescendo, com as profissões que mais evoluem exigindo uma quantidade substancial de interações interpessoais. Suas descobertas apoiam 
um modelo de produção em equipe, em que os trabalhadores trocam tarefas para explorar sua vantagem comparativa.

Nesse cenário, as habilidades sociais reduzem os custos de infraestrutura, permitindo que os funcionários se individualizem e negociem com mais eficiência. Assim, habilidades sociais, como a disposição de cooperar, podem tornar os trabalhadores mais produtivos no trabalho em equipe (Wei, Wang, \& Chen, 2018).

Isso pode ser particularmente importante em ocupações com alta complementaridade entre habilidades cognitivas e sociais. Evidências recentes sugerem que o voluntariado social pode ser usado para sinalizar com credibilidade a disposição de cooperar, mas também pode se correlacionar com outras habilidades tecnológicas valorizadas pelos empregadores (Shukla \& Kumar, 2018; Sudha et al., 2018).

Um estudo observacional sobre o impacto das habilidades na empregabilidade, no entanto, enfrenta o desafio que os pesquisadores não identificam se as habilidades são realmente observadas pelos empregadores. Em contraste com características inatas como gênero e raça, habilidades cognitivas, sociais e maturidade não são diretamente observáveis.

Assim, a empregabilidade pode ser principalmente a função de sinais dessas habilidades, em vez das próprias habilidades tecnológicas (T. Souza, Roncon, \& Lavarda, 2013). Credenciais educacionais, notas e atividades extracurriculares são apenas alguns exemplos proeminentes de tais sinais de habilidade. A literatura também explora o papel dos sinais de habilidades dispendiosos na determinação dos resultados do mercado de trabalho. Estudos investigam o valor de sinalização de credenciais educacionais (Burnier, 2017; Deming, 2017).

A importância dos sinais de habilidade pode, no entanto, depender crucialmente de outros fatores. Por exemplo, parece intuitivo que os sinais de habilidades sejam mais importantes para os que ingressam no mercado de trabalho do que para os trabalhadores com experiência substancial.

De fato, Rifkin (2014) encontra evidências para o aprendizado sobre as verdadeiras habilidades dos trabalhadores ao longo do tempo. Além disso, Lemos (2011) e Rodriguez e Monteiro (2011) mostram que a capacidade é observada de perto para os recém-formados, mas é revelada ao mercado de trabalho mais gradualmente para os concluintes do ensino médio.

\subsection{Habilidades individuais e empregabilidade}

Habilidades individuais são a harmonização entre conhecimentos, competências e atitudes que, uma vez desenvolvidos, proporcionam ao indivíduo e à organização, maiores atributos para competir no mercado em que atua. Assim, com o uso adequado das competências, as tarefas aconteceriam de forma eficaz, contribuindo para a satisfação pessoal (Fongwa, 2018; Porter, 1999). Nesse contexto, essas habilidades determinam um eixo entre a competência dos indivíduos, da equipe e, consequentemente, da organização.

Ao pesquisar as referências para dar suporte teórico à presente pesquisa, foram eleitos quatro componentes principais, os quais incrementam a capacidade do trabalhador atual em termos de empregabilidade.

1. Recursos - referem-se ao conhecimento - o que o indivíduo conhece (Choo, C.; Bontis, 2002; Choo, 2003), habilidades - o que ele pode fazer com o que sabe (Deming, 2017) e atitudes - como ele faz (Raquel \& Ferreira, 2016; Sudha et al., 2018).

2. Desenvolvimento - capacidade de gerenciar a carreira (sabendo quais oportunidades existem e seus requisitos de entrada), capacidade de buscar trabalho (trabalho satisfatório) e abordagem estratégica, ou seja, ser adaptável a eventos de mercado e ser realista sobre oportunidades (Goes \& Pilatti, 2013; Martins \& 
Oliveira, 2016).

3. Apresentação - capacidade de demonstrar os seus recursos (apresentação do curriculum vitae), qualificações individuais, referências e experiências de trabalho (Lysova et al., 2018; M. Souza et al., 2017).

4. Circunstâncias pessoais e o mercado de trabalho - é a habilidade de empregabilidade, que contempla a preocupação com responsabilidades, deficiências pessoais e obrigações familiares capazes de buscar a capacidade de procurar trabalho (Deming, 2017; Rifkin, 2014).

Quanto a competências que o mercado de trabalho exige, quatro principais são mencionados:

1. Valor da informação - como vantagem competitiva envolvendo autoconhecimento, bagagem e experiências pessoais, características que também estão relacionadas à empregabilidade (Edvinsson \& Malone, 1998; Sveiby, 1998).

2. Tecnologia e outras habilidades técnicas - saber como usar tecnologias e outras aplicações (Castells, 2007).

3. Habilidades organizacionais - definir metas e saber administrar o tempo (Porter, 1999; Porto \& Oliveira, 1997).

4. Habilidades decorrentes do processo educativo - a busca constante de informações, o desenvolvimento da escuta e da escrita e a aplicação de tecnologias, talvez sejam mais convergentes com os objetivos deste artigo (Helal, Neves, \& Fernandes, 2007; Martins \& Oliveira, 2016).

\subsection{Marketing Educacional}

O marketing educacional está diretamente ligado às bases e conceitos do marketing de serviços, por isso é importante analisar o mercado e atender o público-alvo. Portanto, deve-se considerar o preço cobrado, o serviço oferecido, se o local atende a sociedade exigente e como será promovido (Kotler, 2017).

Kotler (2009) argumenta que o marketing tem tanto críticos quanto defensores. Alguns educadores sentem que é incompatível com a missão educacional e subestima a educação e as instituições que a utilizam. Por outro lado, seus defensores afirmam que o marketing realmente ajuda a cumprir sua missão educacional, aumentando a satisfação que oferece ao seu mercado-alvo. Para se tornar um candidato ao mercado de trabalho, o educando precisa adquirir um conjunto de habilidades, conhecimentos, conscientização e formação política, ética e cidadã (Borini, 2004, 2008).

O aluno não vai à escola para comprar um produto; o aluno é o produto (Kotler, 2009). É preciso ter qualidade, educação e instrumentos. Atividades, professores, equipamentos e conhecimento são ferramentas para a construção deste produto (Lysova et al., 2018).

Educação acadêmica e profissional não são produtos tangíveis; eles não podem ser vendidos como roupas nas vitrines das lojas. É um processo longo, numa relação entre professor e aluno, que nem sempre é leve e fácil. Não traz satisfação imediata, mas é fundamental para o crescimento e o treinamento dos trabalhadores (Santos, Mesquita, Neves, \& Bastos, 2016).

O objetivo da instituição de formação profissionalizante é aperfeiçoar o trabalhador para ocupar a melhor posição no mercado. Seu cliente não é, portanto, o aluno, mas o mercado de trabalho (Van der Heijden et al., 2018). Além disso, não se pode perder de vista o fato de que o mercado está se tornando mais exigente a cada dia. Assim, contribuir para a empregabilidade do aluno tornará o mercado de trabalho mais satisfatório (Fongwa, 2018; Goes \& Pilatti, 2013). 


\subsection{Modelo Hipotético: principais atributos que podem influenciar a empregabilidade}

Para que as instituições de ensino profissionalizante cumpram sua missão educacional, é importante que todos os seus processos sejam observados, mensurados, aprimorados e executados de maneira eficaz e eficiente.

Assim, após o exame da literatura que permite definir, com mais acuidade, quais apreciações são mais promissoras para se analisar qual a influência dos atributos educacionais dos cursos profissionalizantes na empregabilidade, algumas hipóteses serão propostas, bem como a especificação do campo no qual deve-se dedicar maior atenção (Strauss \& Corbin, 2008).

H1: O Plano Pedagógico influencia positiva e significativamente a empregabilidade.

Plano Pedagógico é o equilíbrio da linha de serviço, em termos de como as diretrizes e prioridades estabelecidas pela equipe da escola serão aplicadas, com base nos objetivos educacionais e na definição dos resultados a serem alcançados, sempre visando melhorar a aprendizagem e o desempenho das equipes (Borini, 2008). Campos (2006) relata a importância de as instituições fornecerem um ensino amplo, flexível e motivador, de forma que o aluno compreenda a importância de seus estudos e do desenvolvimento dessas características e habilidades para melhor exercer suas atividades profissionais.

É muito importante para a instituição remodelar as disciplinas que oferece, no intuito de abarcar as características desejáveis, para a recuperação e o manejo de informações já aprendidas, a comunicação, a apresentação, o planejamento, a resolução de problemas e interação social, e principalmente tornar as aulas mais dinâmicas e atrativas para os alunos. "A educação deve promover habilidades que lhes possibilitem atuar profissionalmente em uma realidade denominada era da aprendizagem ou sociedade de aprendizagem" (Campos, 2006 p. 31).

A importância do Plano Pedagógico tem sido questionada com base em alguns achados experimentais conflitantes. Tomaz (2016) apresentou resultados de pesquisas que possuem como foco os alunos, suas preferências e percepções, que demonstram não ser de grande importância a qualidade dos planos pedagógicos. Para Fongwa (2018), em uma sociedade com tantas desigualdades sociais, qualquer plano pedagógico que enfoque apenas habilidades e empregabilidade sem um foco ativo em questões de cidadania limita o potencial das entidades em contribuir para o desenvolvimento de habilidades humanas.

Em discordância com esses conceitos, Floriano e Hennig (2017) mostram que planos pedagógicos eficientes favorecem o processo de ensino-aprendizagem. Assim, justifica-se a proposição dessa hipótese.

H2: A Infraestrutura influencia positiva e significativamente a empregabilidade.

A instituição educacional precisa tornar seus programas e serviços disponíveis e acessíveis a seus mercados-alvo. Além de organizar as salas e laboratórios com softwares de simulação de rotinas e casos nas áreas de estudo, essas experiências podem contribuir para a empregabilidade do aluno, dando-lhe uma visão real das rotinas e desafios que enfrentará quando confrontado com o mercado de trabalho (Lysova et al., 2018; Faria, 2002).

Campos (2006) sugere que o aluno que busca uma instituição de ensino com o objetivo de aumentar suas chances de empregabilidade é influenciado também por variáveis psicológicas. Aspectos estruturais, como, espaço, iluminação e cores podem influenciar o nível de satisfação, responsabilidade, autoestima e a auto eficácia do aluno no processo de aprendizagem. 
A fim de explicar a importância na proposição desta hipótese, cita-se Floriano e Hennig (2017) que apresentam como resultados de pesquisa que a infraestrutura escolar se constitui como uma das dimensões que determinam a qualidade percebida pelos alunos em serviços educacionais. Por outro lado, Cruz e Azevedo (2012) atestam a existência de um grande número de cursos profissionalizantes sem infraestrutura adequada, que passam uma imagem de oferecer curso técnico que atenda aos requisitos exigidos pelo mercado, embora não satisfaçam condições mínimas.

Embora as instituições de ensino tenham bons diretores, professores dedicados e força de vontade dos alunos, é óbvio que a infraestrutura pode desempenhar um papel fundamental na formação dos alunos, além de garantir conforto e bem-estar não só para os alunos, mas para os professores e toda a comunidade escolar.

H3: O corpo docente influencia positiva e significativamente a empregabilidade.

De acordo com Deming (2017), é possível afirmar que essas condições são menos prováveis de ocorrer em cursos profissionalizantes, mas investigar mostra uma boa alternativa quando tópicos subjetivos, como habilidades, estão sendo pesquisados.

Para que os atributos mencionados funcionem na instituição de ensino, o corpo docente deve ser visto como o maior influenciador no processo de desenvolvimento do aluno e o conhecimento deve ser transmitido visando às questões de empregabilidade (Deming, 2017), diferentemente da visão de Raquel \& Ferreira (2016), para os quais a alta rotatividade entre os jovens docentes poderia impedir uma aproximação entre o corpo docente e os alunos.

Assim, é necessário adotar a postura que o mercado de trabalho exige, a fim de determinar os passos mais eficazes a serem tomadas, de acordo com a fase de formação de cada etapa do aluno, direcionando-o para o personalizado tratamento (Fongwa, 2018). Em posição mais elevada, incluir questões na formação de professores em relação à empregabilidade. De acordo com Faria (2002), oferecer condições dignas de remuneração aos professores e instrutores pela prática da carreira docente contribuirá para motivá-los, e consequentemente, torná-los molas propulsoras dentro da sala de aula, possibilitando o crescimento vertical da instituição e criando uma perspectiva positiva para o aluno que busca a diferenciação em seu papel profissional.

Campos (2006) destaca a importância de oferecer aos professores uma preparação para as necessidades do mercado de trabalho, incluindo-se as novas capacidades, tais como, competência na transmissão das habilidades de carreira, associá-los aos requisitos de ensino e ao trabalho com empresas locais. É de suma importância estabelecer como meta a preparação dos professores, de forma que aprendam a demonstrar as conexões entre o conteúdo teórico de sua área e o mercado de trabalho, aliando, dessa forma, teoria e prática. De acordo com Lynch (2002), para preparar trabalhadores mais qualificados, os professores precisam estar habilitados para unir o ensino com o mercado de trabalho.

H4: As Atividades Extras influenciam positiva e significativamente a empregabilidade.

Esta observação pode apoiar a hipótese de que quaisquer atividades sejam importantes, de modo que contribuam para a visibilidade da instituição de ensino, aumentem sua credibilidade e confiança para o público-alvo. Nesse caminho, destaca-se, conforme Campos (2006):

a) Experiência de liderança na instituição - isso mostra que o aluno está disposto a lidar com as situaçõesproblema e transmite a noção de responsabilidade e gestão de pessoas.

b) Praticar esportes - esse envolvimento transmite o lado competitivo do aluno e o destaca por trabalhar em equipe. 
c) Trabalho voluntário - o interesse fora do campo de estudo revela que o aluno demonstra generosidade, disponibilidade e disciplina. Isso reforça ainda mais a capacidade do aluno de gerenciar projetos, trabalhar com pessoas e melhorar o clima organizacional da empresa.

d) Visitas técnicas - as empresas exercem o papel de formadores de valores no âmbito das relações sociais nas comunidades. Com isso, a instituição consegue vincular seu Plano Pedagógico ao contexto empresarial. A realização desta atividade é de extrema relevância para os alunos que buscam aumentar suas chances de empregabilidade, na medida em que possibilita observar o ambiente real de uma empresa em pleno funcionamento, além de ser possível verificar sua dinâmica, organização e todos os fatores teóricos implícitos nela.

e) Orientação Profissional - serve não apenas para se ter um norte sobre o campo profissional a seguir, mas também como uma oportunidade de autoconhecimento, de alinhamento entre habilidades/características pessoais e profissão, do sentido/significado do trabalho para o ser humano, da relação trabalho e projeto de vida.

Assim, a instituição consegue vincular seu Plano Pedagógico ao contexto empresarial. A realização destas atividades é de extrema relevância para os estudantes que buscam aumentar suas chances de empregabilidade.

Matias (2017) e Mahimuang (2018) afirmam que não há evidências empíricas suficientes para afirmar que atividades extracurriculares possam trazer mais malefícios do que benefícios, mas há indícios de que os efeitos negativos possam aparecer em alguns segmentos.

Perante o desenvolvimento das quatro hipóteses que norteiam o presente estudo, sugere-se omodelo hipotético conforme a Figura 1:

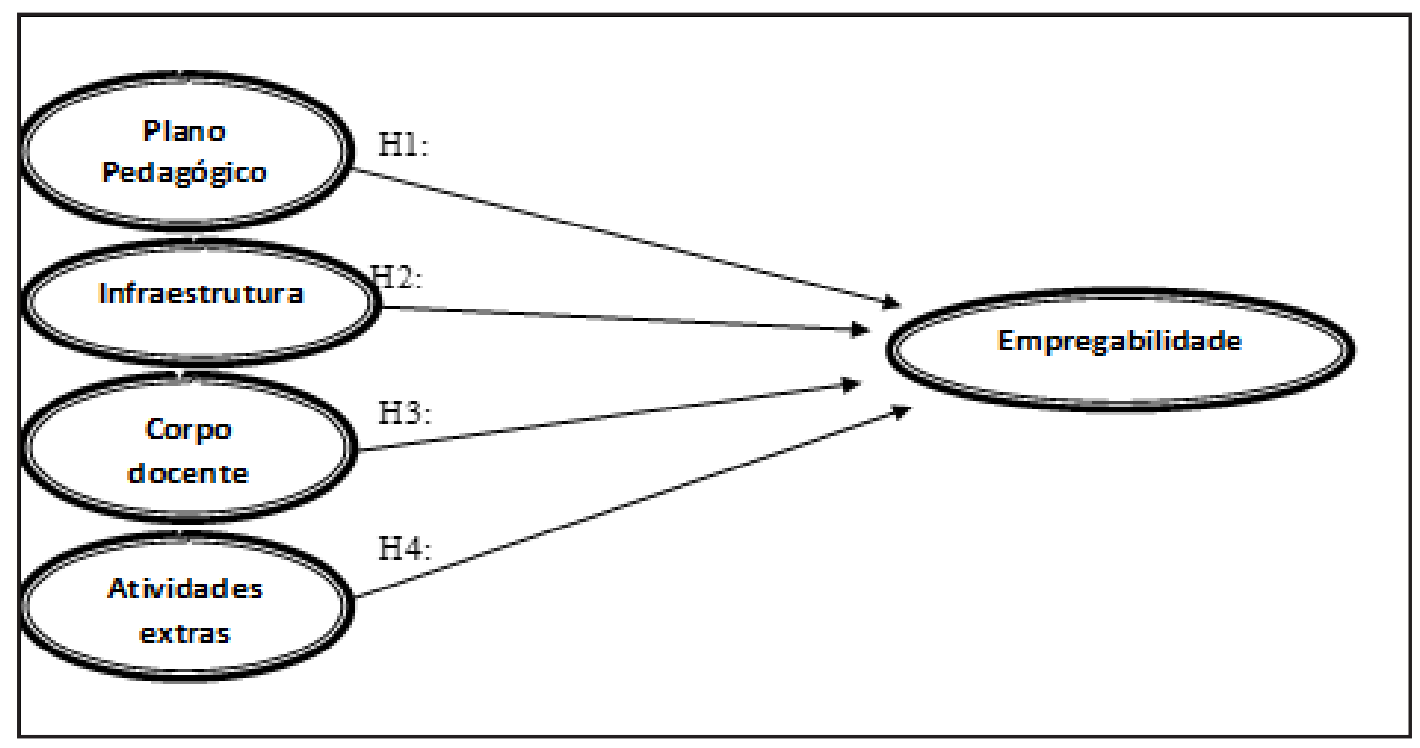

FIG. 1: Modelo hipotético: Os principais fatores que podem influenciar a empregabilidade

\section{Métodos de Pesquisa}

Quanto aos fins, a pesquisa é descritiva, quanto à abordagem, é quantitativa (Venkatesh, Brown, \& Bala, 2013), e foi realizada através de um estudo de caso único (Vergara, 2017; Yin, 2014).

Aamostra foi composta por alunos de uma instituição de ensino profissionalizante, os quais responderam um questionário com 60 perguntas, 9 acerca do perfil sócio demográfico, e 51 questões fechadas, para mensurar os construtos estudados, medidas em uma escala intervalar de 10 pontos, variando de 1 (pouca importância) a 
10 (extremamente importante). Para o fator Plano Pedagógico, foram elaboradas sete perguntas, para o fator Infraestrutura, dez perguntas, para o fator Corpo Docente oito e para o fator Atividades Extras foram também dez perguntas. Ao final da coleta de dados, a amostra foi de cento e quarenta e duas respostas consideradas válidas e respondidas completamente, de um universo total de cento e sessenta respondentes.

A fim de legitimar a análise dos dados (D. Silva, Silva, Braga Junior, Lopes, \& Veiga, 2016) e estimar as relações entre os construtos com base na modelagem de equações estruturais, por meio da estimação por mínimos quadrados parciais empregou-se o software SmartPLS versão 3.0 (RINGLE; WENDE; BECKER, 2015), configurado de acordo com os parâmetros: Weitghting Scheme = Path Weitghting Scheme; Data Metric $=$ Mean 0, Var 1; Data Iterations $=300$; Abort Criterion $=1.0 \mathrm{E}-7$ (isto é, 0,0000001) e Initial Weights $=1.0$ (HAIR JR et al., 2017, p. 91). As cargas fatoriais obtidas após a execução do algoritmo de estimação Partial Least Squares (PLS (Hair, Jr., Hult, Ringle, \& Sarstedt, 2014).

Os achados do trabalho apontaram que compromisso com a qualidade do ensino, ênfase na prática profissional, remodelamento das disciplinas de forma inovadora e conscientização quanto à importância de se capacitar, exercem um efeito positivo e significativo sobre a empregabilidade.

Para mensurar a infraestrutura adotou-se a escala de dez itens desenvolvida por Quintella (2001), considerado um dos principais pilares dos estudos sobre marketing educacional. Os principais itens citados foram: boa localização, excelente infraestrutura, com salas climatizadas e equipadas com datashow, laboratórios de informática bem organizados, softwares para simulações e acesso gratuito à internet, todos essenciais à formação dos alunos, o que contribuirá significativamente para aumentar as chances de empregabilidade do aluno.

Para validar o atributo educacional corpo docente, adotou-se a escala de Martins (2006), com oito itens, destacando-se: professores que adotam a postura do mercado de trabalho no processo de formação, que saibam se relacionar e solucionar problemas, que são um exemplo para os alunos seguirem, motivando-os a aperfeiçoarem suas competências para aumentar a sua chance de empregabilidade.

As atividades extras contribuem para a visibilidade da instituição, sua credibilidade e confiança perante os alunos. Para mensurar o atributo educacional atividades extras, utilizou-se a escala de Martins (2006), a qual possui dez itens. As experiências agregadas aos alunos como, visitas técnicas, prática de esportes, o trabalho voluntário, orientação profissional e programas de estágios, contribuem, positiva e significativamente, para a empregabilidade.

A empregabilidade é vista como algo que motiva as pessoas a se capacitarem. Para mensurar a empregabilidade foi utilizada a escala de 17 itens proposta por Campos (2006). Introduzir no processo educacional conceitos sobre o mercado de trabalho e oportunidades na área de formação e desenvolver a capacidade do aluno de reconhecer suas habilidades e limites, assim como aquelas que ele ainda precisa desenvolver poderão contribuir, positiva e significativamente, para aumentar suas chances de empregabilidade.

De uma amostra de 160 respondentes, foram identificados 18 dados extremos, os quais foram excluídos. Considerando apenas os registros válidos, ou seja 142, os dados demográficos da pesquisa mostram que $29,58 \%$ dos respondentes são do sexo masculino, contra $70,42 \%$ do sexo feminino. $12,68 \%$ dos entrevistados são menores de 18 anos; já 78,87\% têm entre 18 a 40 anos, e outros 8,45\% têm mais de 41 anos. Destaca-se que $100 \%$ dos entrevistados já desenvolveram vários tipos de atividades profissionais. Os dados mostram que $38,71 \%$ possuem experiências na área da educação como professores do ensino fundamental, médio, técnico e superior, 61,29\% nas áreas de Administração, Contabilidade, Design Gráfico, Vendas e Gestão. Verifica-se 
que $25 \%$ dos alunos são formados em contabilidade, por outro lado 18,31\% em Gestão Empresarial e os outros $56,69 \%$ dos alunos possuem formação em áreas específicas, como Design Gráfico, PCP, Vendas Online.

\section{Resultados}

Os dados relativos ao modelo de mensuração são apresentados na Tabela 1. Pelo Coeficiente Alpha de Cronbach (Dirceu da Silva, da Silva, Braga Junior, Luiz Lopes, \& Teixeira Veiga, 2016) e Coeficiente de Confiabilidade Composta (Hair, Jr., Hult, Ringle, \& Sarstedt, 2014) superiores a 0,7, resta atestada a confiabilidade de todos os construtos. A validade convergente foi comprovada pela Variância Média Extraida (AVE) igual ou superior a 0,5 , e pelas cargas fatoriais significativas de todos os indicadores (figura 2). A validade discriminante, pelo o critério de Fornell-Larcker, não foi confirmada apenas no caso dos construtos atividade extras e empregabilidade, mas foi atestada no critério de cargas transversais, razão pela qual prosseguiu-se com a análise.

\section{Tabela 1}

Testes de confiabilidade e validade.

\begin{tabular}{cccccc|ccc}
\hline & AEXT & CDOC & EMPR & INFR & PPED & AC & CC & AVE \\
\hline AEXT & 0,70 & & & & & 0,89 & 0,91 & 0,50 \\
CDOC & 0,70 & 0,75 & & & & 0,89 & 0,91 & 0,56 \\
EMPR & 0,74 & 0,69 & 0,76 & & & 0,95 & 0,96 & 0,58 \\
INFR & 0,58 & 0,56 & 0,45 & 0,72 & & 0,90 & 0,91 & 0,52 \\
PPED & 0,54 & 0,69 & 0,69 & 0,51 & 0,79 & 0,90 & 0,92 & 0,63 \\
\hline
\end{tabular}

Os resultados do modelo estrutural são mostrados na Tabela 2 e Figura 2.

Tabela 2

Modelo estrutural.

\begin{tabular}{ccccc}
\hline Relação & Coeficiente & "t" & Valor p & IC 95\% \\
\hline PPED $>>$ EMPR & 0,3858 & 3,8496 & 0,0002 & {$[0,1241 ; 0,6474]$} \\
INFR $>>$ EMPR & $-0,1211$ & 1,6874 & 0,0937 & {$[-0,3085 ; 0,0663]$} \\
CDOC $>>$ EMPR & 0,1318 & 1,1457 & 0,2539 & {$[-0,1684 ; 0,4320]$} \\
AEXT $>>$ EMPR & 0,5101 & 5,1548 & 0 & {$[0,2515 ; 0,7686]$} \\
\hline
\end{tabular}

Das hipóteses propostas, duas foram rejeitados (H2: Infraestrutura influência positiva e significativamente a empregabilidade, H3: Corpo docente influência positiva e significativamente a empregabilidade) e as outras duas confirmadas (H1: Plano pedagógico influenciainfluência positiva e significativamente a empregabilidade, H4: Atividades extras influenciam positiva e significativamente a empregabilidade).

A hipótese "A Infraestrutura influência positiva e significativamente a empregabilidade", mesmo que estatisticamente significativa, é rejeitada, pois indica um efeito negativo da infraestrutura na empregabilidade $(<0,10)$. De acordo com David e Saeipoor (2018) Lysova et al., 2018; Floriano e Hennig (2017) e Faria, 2002, a instituição educacional deve disponibilizar seus programas e serviços e torná-los acessíveis aos seus mercados-alvo. Os autores apontam que, além de organizar as salas e laboratórios com softwares para simulação de rotinas e casos nas áreas de estudo, essas experiências podem contribuir para a empregabilidade do aluno.

Assim, a pesquisa mostra que esses fatores não influenciam o aluno na escolha da instituição de ensino, que nem sempre olham para as condições físicas e estruturais. Gandini (2015) sugere que o aluno é influen- 
ciado por variáveis de impacto cognitivo como espaço, iluminação e cores durante o processo de formação, entretanto, como a Infraestrutura não desempenha papel fundamental na formação dos alunos, eles não consideram tais fatores decisivos para empregabilidade. Cruz e Azevedo (2012) afirmam haver escolas que não oferecem condições adequadas, o que pode ter influenciado a rejeição da hipótese.

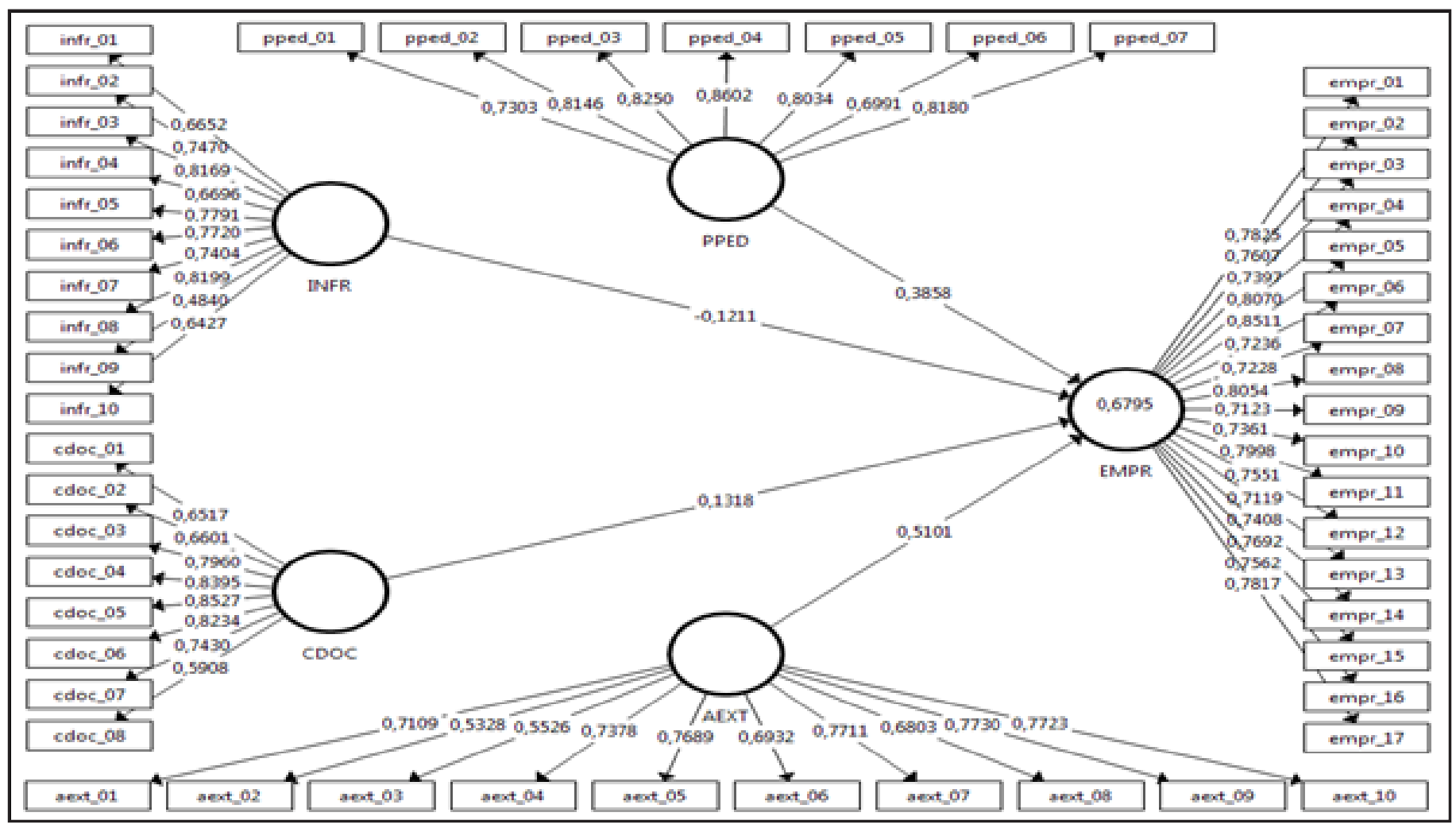

Figura 2. Modelo Estrutural. Resultados do algoritmo PLS

A hipótese "O corpo docente influencia positivamente e significativamente a empregabilidade" é rejeitada, uma vez que a relação proposta não é estatisticamente significativa. Embora o corpo docente seja percebido como o principal fator de influência no processo de formação, os estudantes na contratação de tais serviços, já esperam qualidade e bom atendimento dos serviços prestados e que suas expectativas sejam superadas.

A pesquisa apontou que o corpo docente não influencia significativamente a empregabilidade do aluno, pois, do ponto de vista deles, é obrigação da instituição ter os melhores profissionais do ensino e oferecer treinamento para os professores sobre como ensinar habilidades de carreira. Para Raquel \& Ferreira (2016), a rotatividade de docentes pode comprometer a sua relevância como atributo capaz de aumentar a empregabilidade, fato que não foi mensurado na pesquisa, mas talvez ajude a justificar a rejeição da hipótese.

As hipóteses H1: Plano pedagógico influência positiva e significativamente a empregabilidade, H4: As atividades extras influenciam positiva e significativamente a empregabilidade são suportadas pelo modelo estrutural.

De acordo com Faria (2002), o Plano Pedagógico é o equilíbrio da linha de serviços, de como serão aplicadas as diretrizes e prioridades estabelecidas pela equipe escolar, a partir dos objetivos educacionais e da definição dos resultados a serem atingidos. "A educação deve promover habilidades que lhes possibilitem atuar profissionalmente em uma realidade denominada era da aprendizagem ou sociedade de aprendizagem" (Campos, 2006 p. 31). 
Floriano \& Henning, (2017) e Campos (2006) ressaltam a importância da complementação do ensino de sala de aula com a aprendizagem da realidade de trabalho, no intuito de ajudar os alunos a combinarem suas compreensões teóricas com a aplicação prática para assim, estarem bem mais preparados para enfrentar o mercado de trabalho. Tudo se deve a um Plano Pedagógico bem elaborado, que esteja de acordo com a missão da instituição de ensino que seja aplicado dentro das salas de aulas.

Os resultados apontam que as atividades extras também influenciam, positiva e significativamente, a empregabilidade do aluno. Campos (2006) afirma que a adoção de programas de estágios, visitas técnicas, incentivo à produção de oficinas, workshops, programas de orientação profissional e encaminhamento profissional, entre outros serviços, contribuirá para o aumento da prática/experiência exigida pelo mercado de trabalho e para a quebra de barreiras dos alunos em questões de ordem pessoal, social e técnica. Segundo Lemos \& Costa (2012), as instituições de ensino devem estar atentas às novas formas de utilização de tecnologias auxiliares para levar seus serviços aos seus clientes, a fim de contribuir efetivamente para a aprendizagem do aluno.

Ao aliar os conceitos teóricos estudados na sala de aula à prática, as chances de empregabilidade do egresso aumentam consideravelmente. Promover experiências baseadas no desenvolvimento de habilidades mentais gerais, como, responsabilidade, brilhantismo, agilidade na solução de problemas e facilidade em aprender novas habilidades, de acordo com Campos (2006), deve constituir-se em um dos pontos mais utilizados pelos selecionadores no momento de contratar uma pessoa.

Faria (2002) conclui que maior conhecimento e vivência sobre o mercado de trabalho podem auxiliar o aluno a encontrar melhores colocações profissionais, por aumentarem as suas condições de empregabilidade.

De acordo com as cargas fatoriais, verifica-se que algumas das dependências foram relacionadas positivamente e foram estatisticamente significantes, de modo que o construto empregabilidade obteve um valor de $\mathrm{R}^{2}=0,6795$.

Isso significa que, como detalhado na sessão de referências, os construtos plano pedagógico, infraestrutura, corpo docente e atividades extras foram capazes de explicar a variação correspondente a um valor observado em $68 \%$ da empregabilidade. O poder preditivo do modelo é elevado, $\mathrm{Q}^{2}=0,3602$.

\section{Considerações finais (contribuição para ciência, para academia e para o mercado)}

O marco teórico permitiu vislumbrar uma série de características tidas como necessárias para que uma pessoa se torne empregável. As instituições de ensino, com base nesses pressupostos, começaram a implantar ações voltadas a aumentar a empregabilidade dos alunos. É nesse contexto que se enquadra esta pesquisa. No mundo do trabalho surgem mudanças nos padrões de comportamento das pessoas e das empresas, decorrendo daí a necessidade de criar instrumentos que acompanhem tal evolução.

Pelos resultados, foi possível perceber a conexão de cada um dos construtos propostos com a empregabilidade. Após a análise estatística, verificou-se que das quatro hipóteses propostas duas foram confirmadas e duas foram rejeitadas.

Os fundamentos que compõem as duas hipóteses confirmadas foram: Plano Pedagógico e Atividades Extras. No Marco Teórico, argumentou-se que ambos podem criar valor e aumentar a empregabilidade.

No entanto, as relações entre os construtos Infraestrutura e Corpo Docente com o construto Empregabilidade não são estatisticamente significantes. Tais resultados conflitam com a argumentação teórica.

Cabe agora ressaltar as limitações e contribuições da pesquisa, assim como tecer sugestões para futuras inves- 
tigações. O estudo não pode ser generalizado, pois se trata de um estudo de caso único, com base na percepção dos próprios alunos do caso investigado. Ainda, a pesquisa teve como foco a análise do ambiente externo, não contemplando os demais stakeholders, como funcionários e fornecedores, que poderiam contribuir para alterar os resultados do estudo. Outro ponto de limitação prende-se ao fato de não se ter objetivado analisar os elementos capazes de influenciar o resultado segmentado por curso, além de não ter sido considerada a possibilidade do surgimento de novos construtos que poderiam medir o nível de empregabilidade dos alunos.

Como contribuições acadêmicas, pode-se elencar a análise de elementos amplamente abordados na literatura específica, mas de forma isolada. A conjugação dos construtos em um único modelo conceitual mostrase inovadora e permitiu avaliar a influência de cada um sobre a empregabilidade. Tal contribuição permitirá a evolução de análises, o aprofundamento nos elementos propostos e a ampliação do estudo a outros construtos.

Ainda, pode-se considerar como benefício deste trabalho para a literatura sobre gestão educacional, a oferta de um instrumento de pesquisa validado, o qual pode subsidiar a realização de outros estudos relativos ao tema.

Para as instituições de ensino, a principal contribuição é o fato de o modelo conceitual proposto poder ser visto como base de referência para guiar a organização rumo a um melhor desempenho, isso considerando que a pesquisa explicita elementos que estatisticamente foram aceitos como significativos em relação à empregabilidade. Dessa forma, gestores das instituições de ensino profissionalizantes podem considerar os elementos significativos da pesquisa para direcionar o rumo de suas organizações que terão maiores chances de êxito em suas decisões no processo educacional.

Outra contribuição é observar o fato de que a pesquisa alerta para que mesmo elementos considerados como chaves numa estratégia de marketing educacional podem ser comprometidos pelo contexto no qual eles estão sendo aplicados.

Considerando que em nenhum momento houve a intenção de esgotar a possibilidade de estudos relacionados ao tema pesquisado, recomendam-se para a realização de trabalhos futuros alguns temas. A pesquisa considerou uma perspectiva transversal - ou seja, a extração da percepção do momento - mas referente a informações longitudinais - ou seja, fatos ocorridos ao longo de um tempo determinado. Nesse sentido, indica-se a aplicação da pesquisa em um novo momento, ampliando o período de análise.

Outra possibilidade seria realizar a pesquisa com o intuito de analisar a influência dos elementos em cada construto, além de identificar outros construtos que não foram utilizados nessa pesquisa.

Por fim, mostra-se relevante avaliar os construtos Infraestrutura e Corpo Docente, os quais não apresentaram resultados significativos na pesquisa, a despeito de toda uma base teórica que realça a sua importância na empregabilidade.

Diversos autores reforçam que a estrutura física das escolas pode oferecer subsídios que influenciam não apenas em ganhos da capacidade cognitiva e motora, mas também de socialização dos alunos. Ademais, indicam que, em uma instituição de ensino, o papel da equipe de professores é identificar as expectativas e necessidades dos seus alunos e atendê-las, contribuindo para o seu crescimento pessoal, acadêmico e profissional.

Assim, é interessante ampliar as pesquisas para entender o cenário criado na mente dos alunos, de forma a entender a rejeição dessas hipóteses, ou vir a confirma-las em outros cenários. 


\section{Referências}

Arraes, V., Cabral, A., Santos, S., Silva, M., \& Penha, E. (2017). Relação Entre Aspectos De Modernidade Organizacional E Comprometimento Organizacional. Revista FACES, 16(1), 66-85. https://doi.org/http:// dx.doi.org/10.21714/1984-6975FACES2017V16N1ART3759

Bernstein, J., Machlup, F., Polanyi, M., \& Shera, J. (2014). Disciplinarity and trandisciplinarity in the study of knowledge. Informing Science, 17, 241-273. Retrieved from http://academicworks.cuny.edu/kb_pubs

Borini, F. M. (2004). Mecanismos de Transferência de Conhecimento em Multinacionais Emergentes: survey com as multinacionais brasileiras., 1-16.

Borini, F. M. (2008). Mecanismos de Transferência de Conhecimento praticados por Multinacionais: estudo comparativo entre Multinacionais Tradicionais e Emergentes. Anais ENANPAD - XXV Simpósio de Gestão Da Inovação Tecnológica, 1-16.

Burnier, S. (2017). Projetos de trabalhadores: os significados dos cursos profissionalizantes, 1-7.

Castells, M. (2007). Communication , Power and Counter-power in the Network Society, 1(June 2006), 238266.

Choi, B., \& Pak, A. (2006). Multidisciplinarity, interdisciplinarity and transdisciplinarity in health research and policy. Clinical and Investigative Medicine, 29(6), 351-364. https://doi.org/10.1002/eji.201090065

Choo, C.; Bontis, N. (2002). Intellectual Capital. In OXFORD \& U. PRESS (Eds.), The Strategic Management of Intellectual Capital and Organizational Knowledge.

Choo, C. (2003). A Organização do Conhecimento. (Senac, Ed.) (4th ed.). São Paulo: Senac.

Da Silva, Dirceu, da Silva, A. M., Braga Junior, S. S., Luiz Lopes, E., \& Teixeira Veiga, R. (2016). AVALIAÇÃO DA QUALIDADE PERCEBIDA DE CURSOS GESTÃO EM NÍVEL DE GRADUAÇÃO NA MODALIDADE EAD. ASSESSMENT OF PERCEIVED QUALITY IN THE DISTANCE LEARNING MANAGEMENT UNDERGRADUATECOURSES., 14(1), 242-268. Retrieved from http://10.0.60.240/16795350/rau.v14n1p242-268

Deming, D. (2017). The Growing Importance of Social Skills in the Labor Market. Quarterly Journal of Economics.

Edvinsson, L., \& Malone, M. (1998). Intellectual capital : realizing your company's true value by finding its hidden brainpower. HarperBusiness.

Floriano, M., \& Henning, A. (2017). Endomarketing em instituições de ensino superior: um estudo 
na Universidade Federal do Pampa. XVII Colóqiuio Internacional de Gestão Universitária. - Mar Del Plata. Retrieved from https://repositorio.ufsc.br/xmlui/bitstream/handle/123456789/181087/101_00159. pdf? sequence $=1$ \&isAllowed $=\mathrm{y}$

Fongwa, S. (2018). Higher education 's contribution to graduate employability and the social good, 114(1), $2-3$.

Goes, P. B. de, \& Pilatti, L. A. (2013). Theoretical Interinfluência of Vocational Training and Skills Employability. Nucleus, 10(2), 57-70. https://doi.org/10.3738/1982.2278.834

Helal, D., Neves, J., \& Fernandes, D. (2007). E mpregabilidade Gerencial no Brasi Brasil Diogo Henrique Helal. ANPAD - RAC-Eletrônica, v. 1, n. 2, Art. 1, p. 1-19, Maio/Ago., 1-19.

Kotler, P. (2009). Administração de Marketing: Análise, Planejamento, Implementação e Controle - 5 ed. São Paulo: Atlas, 2008.

Kotler, P. (2017). Philip Kotler: some of my adventures in marketing. Journal of Historical Research in Marketing, 9(2), 203-208. https://doi.org/10.1108/JHRM-11-2016-0027

Lemos, H., \& Costa, A. (2012). A Dimensão Simbólica da Empregabilidade: Mercado, Políticas Públicas e Organização Social do Trabalho. Sociedade, Contabilidade e Gestão, 7(2), 85-103.

Lemos, H., Rocha-Pinto, S., \& Dubeux, V. (2014). College Degree, Insertion in the Job Market and Social Background., 48-64.

Lemos, H., Rodriguez, D., \& Monteiro, V. (2011). Empregabilidade e sociedade disciplinar: uma análise do discurso do trabalho contemporâneo à luz de categorias foucaultianas. Organizações \& Sociedade, 18(59), 587-604. https://doi.org/10.1590/S1984-92302011000400002

Lysova, E. I., Jansen, P. G. W., Khapova, S. N., Plomp, J., \& Tims, M. (2018). Examining calling as a doubleedged sword for employability. Journal of Vocational Behavior, 104(November 2017), 261-272. https://doi. $\operatorname{org} / 10.1016 /$ j.jvb.2017.11.006

Ma, L., Liu, Z., Jiang, M., Yu, K., \& Gan, J. (2017). A Study on regional innovation policy under innovation paradigm 3.0: A case of Jiangsu Province in China. In PICMET 2016 - Portland International Conference on Management of Engineering and Technology: Technology Management For Social Innovation, Proceedings (pp. 1055-1064). https://doi.org/10.1109/PICMET.2016.7806596

Martins, B. V., \& Oliveira, S. R. de. (2016). A Relação entre Formação Profissional e Empregabilidade: em Questão os Jovens Provenientes de Cursos Superiores de Tecnologia. XL Encontro Da ANPAD, 1-15. 
Moriset, B. (2019). Building new places of the creative economy: The rise of coworking spaces. SSRN Electronic Journal, 24.

Porter, M. (1999). Competição - Estratégias Competitivas Essenciais. (Kindle, Ed.) (1st ed.). New York: Kindle.

Porto, A. \& Oliveira, G. D. De. (1997). Gestão do Conhecimento nas Escolas Técnicas Profissionalizantes: as Escolas Ensinam, mas Como Será que Aprendem?, 1-14.

Raquel, S., \& Ferreira, M. (2016). Aa caracterísiticas comportamentais empreendedoras e o desempenho individual : Um Estudo na Indústria Criativa da Publicidade. Revista Perspectivas Contemporâneas, 84-106.

Rifkin, J. (2014). The End of Work. The Decline of the Global Labor Force and the Dawn of the Post-Market Era. Journal of Leisure Research.

Santos, P. R. dos, Mesquita, J. M. C. de, Neves, J. T. de R., \& Bastos, A. M. (2016). Inserção no mercado de trabalho e a empregabilidade de bacharéis em Biblioteconomia TT - Insertion in the labor market and the employability of graduates in Library. Perspectivas Em Ciência Da Informação, 21(2), 14-32. https://doi. org/10.1590/1981-5344/2563

Shukla, A., \& Kumar, S. (2018). An Analytical Study on Stress among Students of Professional Courses in Kanpur Region. Swaranjali Publication, 183-189.

Silva, D., Silva, A. M., Braga Junior, S. S., Lopes, E. L., \& Veiga, R. T. (2016). Avaliação da Qualidade Percebida de Cursos Gestão em Nível de Graduação na Modalidade EAD. Revista de Administração Da Unimep, 14(1), 242-268. https://doi.org/10.15600/1679-5350/rau.v14n1p242-268

Snihur, Y., \& Wiklund, J. (2018). Searching for innovation: Product, process, and business model innovations and search behavior in established firms. Long Range Planning, (May), 1-21. https://doi.org/10.1016/j. $\operatorname{lrp} .2018 .05 .003$

Souza, M., Helal, D., \& Paiva, K. (2017). Burnout e jovens trabalhadores, 751-763.

Souza, T., Roncon, A., \& Lavarda, C. (2013). Gerenciamento da estratégia de uma empresa de cursos profissionalizantes à luz das alavancas de controle de Simons. ANAPAD - VI Encontro de Estudos Em Estratégia, 1-15. Retrieved from http://www.anpad.org.br/diversos/trabalhos/3Es/3es_2013/2013_3Es132. pdf

Stewart, T. A. (1998). The wealth of knowledge : intellectual capital and the twenty-first century organization. Crown Business. Retrieved from https://books.google.com.br/books?hl=pt-BR\&lr=\&id=7fNJmYhD36sC\&o $\mathrm{i}=$ fnd\&pg $=$ PA3\&dq=thomas + stewart + intellectual + capital\&ots=QSEbmbziud\&sig=JZ4PlyaQGBAP6CIH84 
GcGbE_3Ys\#v=onepage\&q=thomas stewart intellectual capital\&f=false

Strauss, A., \& Corbin, J. (2008). Pesquisa Qualitativa: Tipos Fundamentais. RAE - Artigos, 20-29.

Sudha, B., Samuel, A. J., \& Narkeesh, K. (2018). Feasibility online survey to estimate physical activity level among the students studying professional courses: A cross-sectional online survey. Journal of Exercise Rehabilitation, 14(1), 58-63. https://doi.org/10.12965/jer.1835130.565

Sveiby, K. (1998). A knowledge-based theory of the firm to guide in strategy formulation. Journal of Intellectual Capital, 2(4), 344-358. https://doi.org/10.1108/14691930110409651

Van der Heijden, B. I. J. M., Notelaers, G., Peters, P., Stoffers, J. M. M., De Lange, A. H., Froehlich, D. E., \& Van der Heijde, C. M. (2018). Development and validation of the short-form employability five-factor instrument. Journal of Vocational Behavior, 106(March 2017), 236-248. https://doi.org/10.1016/j.jvb.2018.02.003

Venkatesh, V., Brown, S., \& Bala, H. (2013). Bridging the Qualitative-Quantitative Divide: Guidelines for Conducting Mixed Methods Research in Information Systems. Management Information Systems Quarterly, 37(3), 855-879.

Vergara, S. (2017). Análise de conteúdo como técnica de análise de dados qualitativos no campo da administração: potencial e desafios. Revista de Administração Contemporânea, 15(4), 761-765. https://doi. org/10.1590/S1415-65552011000400012

Wei, W., Wang, J., \& Chen, X. (2018). Psychological contract model for knowledge collaboration in virtual community of practice: An analysis based on the game theory. Applied Mathematics and Computation Volume 329.

Yin, R. K. (2014). Estudo de caso - planejamento e métodos. Porto Alegre: Bookman.

Zanini, M. T. (2007). Empirical Analysis: Hypotheses and Methods. In Trust within Organizations of the New Economy (pp. 161-212). Wiesbaden: Deutscher Universitätsverlag. https://doi.org/10.1007/978-3-83505410-3_4

Zobel, A. (2017). Benefiting from Open Innovation: A Multidimensional Model of Absorptive Capacity. Journal of Product Innovation Management, 34(3), 269-288. https://doi.org/10.1111/jpim.12361 